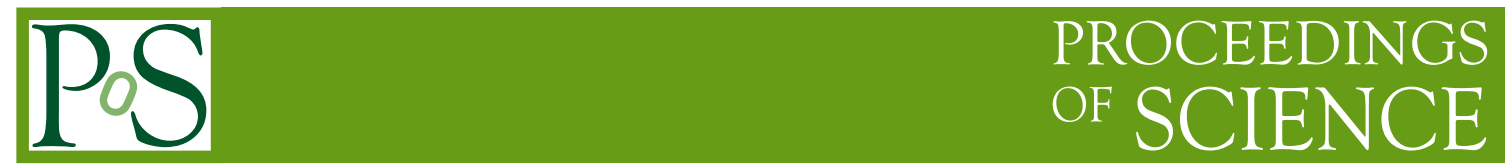

\title{
Charmed Hadron Interactions
}

\author{
Liuming Liu* \\ Department of Physics, College of William and Mary, Williamsburg, VA, 23187, USA \\ Jefferson Laboratory, Newport News, VA, 23606, USA \\ E-mail: lxliux@wm.edu
}

We calculate the scattering lengths of the scattering processes where one or both hadrons contain charm quarks in full lattice QCD. We use relativistic Fermilab formulation for the charm quark. For the light quark, we use domain-wall fermions in the valence sector and improved KogutSusskind sea quarks. In $J / \Psi-N$ and $D-K$ channels, we observe attractive interactions. In $D-D^{*}$ channel, the sign of the scattering length changes, which suggests a bound state.

The XXVII International Symposium on Lattice Field Theory

July 25-31 2009

Beijing, China

${ }^{*}$ Speaker. 


\section{Introduction}

Lattice QCD calculations of the properties of hadronic interactions such as elastic scattering phases shifts and scattering lengths have recently started to develop. Precision results have been obtained in the light meson sector for certain processes such as pion-pion, kaon-kaon and pionkaon scattering and preliminary results for baryon-baryon scattering lengths have been presented. A review of these calculations can be found in [1]. In the heavy meson sector, only a few quenched calculation have been done [2,3]. In this work we study scattering processes where one or both hadrons contain charm quarks in full lattice QCD.

In $2003 \mathrm{BaBar}$ Collaboration discovered a positive-parity scalar charm strange meson $D_{S J}(2317)$ with a very narrow width. CLEO Collaboration confirmed this state later. The discovery of this state has inspired heated discussion in the past several years. The key point is to understand the low mass of this state. There are several interpretations of its structure, such as being, a $D K$ molecule, the chiral partner of $D_{s}$, a conventional $c \bar{s}$ state, coupled-channel effects between the $c \bar{s}$ state and $D K$ continuum etc. See reference [4] for a detailed review. The study of $D K$ interaction is very important in understand the structure of $D_{s J}(2317)$.

The study of the interaction of charmonium and nucleon is also very interesting. As it has been pointed out in the literature [5, 6, 7], such interaction has a direct relation to possible charmoniumnucleus bound states with binding energy of a few $\mathrm{MeV}$. Unlike the traditional nuclear force that binds nucleons, in this case, there are no quark exchange diagrams, and only gluons are responsible for the binding. In other words, the charmonium nucleon force is purely a gluonic van der Waals force.

A number of hadronic states which can not be easily accommodated in the conventional quark model have been observed experimentally in recent years, such as $X(3872), Y(4260), Z^{+}(4430)$. See reference [4] for a detailed review. They are possible candidates of the molecular states composed of two $D$ mesons. These loosely bound states are formed by exchanging light mesons. The mass of $X(3872)$ is very close to the threshold of $D \bar{D}^{*}$. In this work we study the $D \bar{D}^{*}$ interaction. Our result shows that there is very likely a bound state in this channel.

Extracting hadronic interactions from Lattice QCD calculations is not straightforward due to the Maiani-Testa theorem [8], which states that the S-matrix can not be extracted from infinitevolume Euclidean-space Green functions except at kinematic thresholds. However, this problem can be evaded by computing the correlation functions at finite volume. Lüscher has shown that one can obtain the scattering amplitude from the energy of two particles in finite volume $[9,10]$. We use Lüscher's finite volume technique to calculate the scattering lengths.

\section{Lattice Formulaiton}

In this work we employ the "coarse" $(a \simeq 0.125 \mathrm{fm})$ gauge configurations generated by the MILC Collaboration using the one-loop tadpole-improved gauge action. For the fermions in the vacuum, the asqtad-improved Kogut-Susskind action is used. For the valence light quarks (up, down and strange) we use the five-dimensional Shamir domain-wall fermion propagators calculated by the NPLQCD Collaboration. For the charm quark we use the Fermilab action, which controls 
discretization errors of $\mathscr{O}\left(\left(a m_{Q}\right)^{n}\right)$. For the details of the configurations and the quark actions, see our previous work [11] and the references there.

\section{Lüscher's formula}

Lüscher has shown that the scattering phase shift is related to the energy shift $(\Delta E)$ in the total energy of two interacting hadrons in a finite box $[9,10]$.

The center-of-mass momentum $p$ can be obtained by the relation

$$
\Delta E=\sqrt{m_{1}^{2}+p^{2}}+\sqrt{m_{2}^{2}+p^{2}}-m_{1}-m_{2},
$$

where $m_{1}$ and $m_{2}$ are the rest masses of the two hadrons.

To obtain $p \cot \delta(p)$, where $\delta(p)$ is the phase shift, we use the formula

$$
p \cot \delta(p)=\frac{1}{\pi L} \mathbf{S}\left(\left(\frac{p L}{2 \pi}\right)^{2}\right)
$$

where the $\mathbf{S}$ function is defined as

$$
\mathbf{S}(x)=\sum_{\mathbf{j}}^{|\mathbf{j}|<\Lambda} \frac{1}{|\mathbf{j}|^{2}-x}-4 \pi \Lambda .
$$

The sum is over all three-vectors of integers $\mathbf{j}$ such that $|\mathbf{j}|<\Lambda$, and the limit $\Lambda \rightarrow \infty$ is implicit.

If the interaction range is much smaller than the lattice size, $p \cot \delta(p)$ will be

$$
p \cot \delta(p)=\frac{1}{a}+\mathscr{O}\left(p^{2}\right)
$$

where $a$ is the scattering length. The higher order terms in Eq. 3.4 can be ignored if the effective range of the interaction is much smaller than the length scale associated to the center of mass momentum $p$.

From Eq. 3.2 and Eq. 3.4, we get the scattering length to be

$$
a=\frac{\pi L}{\mathbf{S}\left(\left(\frac{p L}{2 \pi}\right)^{2}\right)} \text {. }
$$

\section{Charmed Hadron Interactions: Numerical Results}

In the following, we list all the channels we study. The interpolating operators for these two particle states are composed by the one particle state operators.

$$
\begin{array}{rcc}
\mathscr{O}_{\eta_{c}-\pi}(t)=\eta_{c}(t) \pi^{+}(t) & \mathscr{O}_{J / \Psi-\pi}(t)=J / \Psi(t) \pi^{+}(t) & \mathscr{O}_{\eta_{c}-N}(t)=\eta_{c}(t) N(t) \\
\mathscr{O}_{J / \Psi-N}=J / \Psi(t) N(t) & \mathscr{O}_{D_{s}-\pi}=D_{s} \pi^{+} & \mathscr{O}_{D-\pi}^{I=1}=D^{+} \pi^{+} \\
\mathscr{O}_{D-\bar{K}}^{I=1}=D^{+} \bar{K}^{0} & \mathscr{O}_{D-\bar{K}}^{I=0}=D^{+} K^{-}-D^{0} \bar{K}^{0} & \mathscr{O}_{D-K}^{I=1}=D^{+} K^{+} \\
\mathscr{O}_{D_{s}-K}=D_{s}^{+} K^{+} & \mathscr{O}_{D-1}^{I=\bar{D}^{*}}=D^{+} \bar{D}^{0^{*}} &
\end{array}
$$



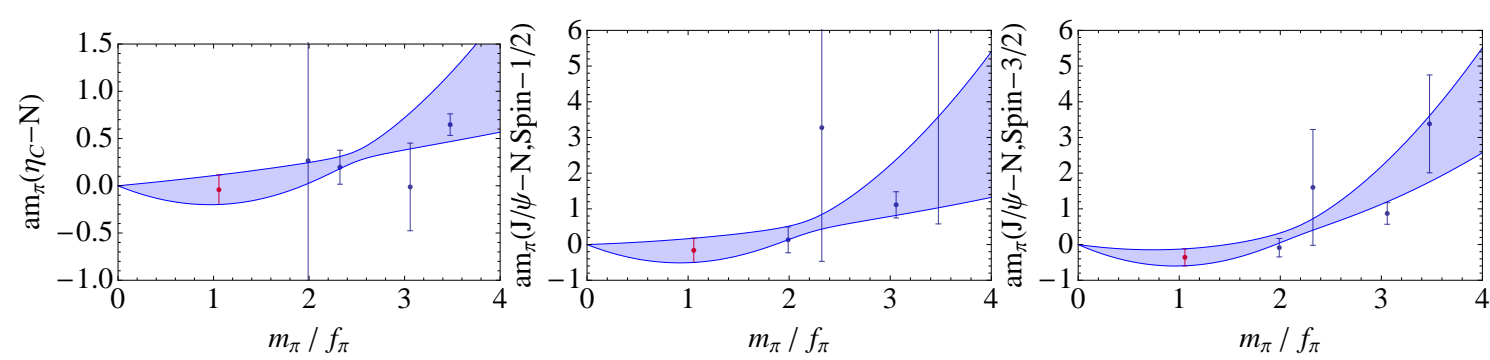

Figure 1: Scattering lengths of $\eta_{c}-N$ and $J / \Psi-N$ as functions of $m_{\pi} / f_{\pi}$. The blue points are our data points. The red points are extrapolated values. The blue shades show the standard deviation allowed region of the fits.

The total energy of two interacting hadrons $\left(h_{1}\right.$ and $\left.h_{2}\right)$ is obtained from the four-point correlation function:

$$
G^{h_{1}-h_{2}}(t)=\left\langle\mathscr{O}_{h_{1}-h_{2}}(t)^{\dagger} \mathscr{O}_{h_{1}-h_{2}}(0)\right\rangle .
$$

To extract the energy shift $\triangle E$, we define a ratio $R^{h_{1}-h_{2}}(t)$ :

$$
R^{h_{1}-h_{2}}(t)=\frac{G^{h_{1}-h_{2}}(t)}{G^{h_{1}}(t) G^{h_{2}}(t)} \longrightarrow \exp (-\triangle E \cdot t)
$$

where $G^{h_{1}}(t, 0)$ and $G^{h_{2}}(t, 0)$ are two-point functions. $\triangle E$ is obtained by fitting $R^{h_{1}-h_{2}}(t)$ to a single exponential.

\subsection{Charmonium-Nucleon}

To extrapolate the scattering lengths of charmonium-nucleon, we perform simple polynomial fits.

$$
a m_{\pi}=c_{1} \frac{m_{\pi}}{f_{\pi}}+c_{2} \frac{m_{\pi}^{2}}{f_{\pi}}
$$

Fig. 1 shows the extrapolations. Our results show that the interaction of $\eta_{c}$ and nucleon is very weak. For the $J / \Psi-N$ channels our data points generally show an attractive interaction, but the extrapolated scattering lengths to the physical pion mass point are very close to zero.

4.2 $D-K(\bar{K})$

The scattering lengths of heavy mesons and light psudoscalar mesons have been studied in chiral perturbation theory. [12, 13]. For simplicity, we use the polynomial functions inspired by the $\chi$ PT to do the extrapolation. Expanding the scattering length in $m_{K}$, we have

$$
a=\left(1+\frac{m_{K}}{m_{D}}\right)^{-1}\left(c_{1} \frac{m_{K}}{f_{K}^{2}}+c_{2} \frac{m_{K}^{2}}{f_{K}^{2}}+\cdots\right) .
$$

For the $D-K, I=1$ channel the first term vanish, we choose the dimensionless fit function

$$
a \frac{m_{K}^{2}}{\mu}=c_{3} \frac{m_{K}^{3}}{f_{K}^{3}}+c_{4} \frac{m_{K}^{4}}{f_{K}^{4}}
$$



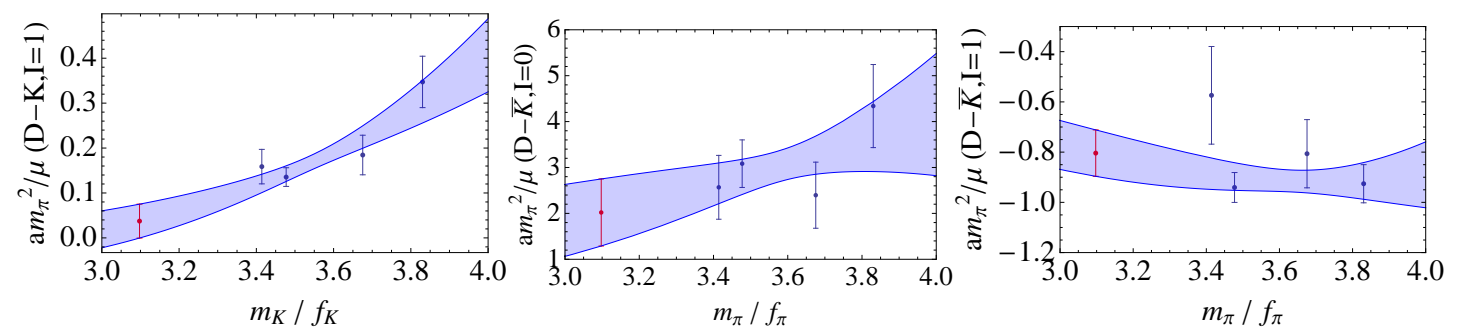

Figure 2: Light quark extrapolation of the scattering lengths of $D-K$ and $D-\bar{K}$. The blue points are our data points. The red points are extrapolated values. The blue shades show the standard deviation allowed region of the fits.

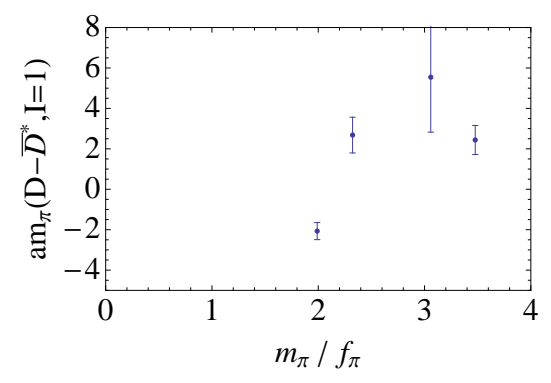

Figure 3: Scattering lengths of $D-\bar{D}^{*}(I=1)$ as a function of $m_{\pi} / f_{\pi}$.

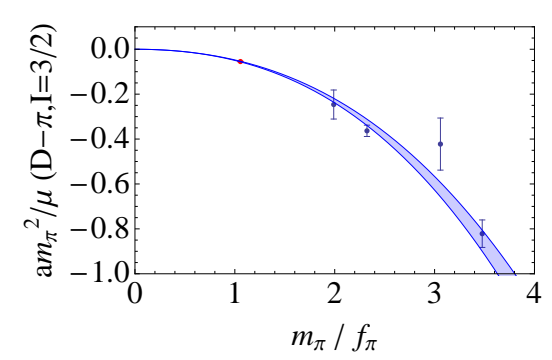

Figure 4: Scattering length of $D-\pi$ spin $3 / 2$ as a function of $m_{\pi} / f_{\pi}$.

where $\mu$ is the effective mass $\mu=\frac{m_{D} m_{K}}{m_{D}+m_{K}}, c_{3}$ and $c_{4}$ are the coefficients to be fitted.

For the $D-\bar{K}$ channels the coefficients of the first terms are fixed by the $\chi$ PT. We choose the fit functions

$$
\begin{aligned}
a^{(I=1)} \frac{m_{K}^{2}}{\mu} & =-\frac{1}{8 \pi} \frac{m_{K}^{2}}{f_{K}^{2}}+c_{3}^{\prime} \frac{m_{K}^{3}}{f_{K}^{3}}+c_{4}^{\prime} \frac{m_{K}^{4}}{f_{K}^{4}} \\
a^{(I=0)} \frac{m_{K}^{2}}{\mu} & =\frac{1}{8 \pi} \frac{m_{K}^{2}}{f_{K}^{2}}+c_{3}^{\prime \prime} \frac{m_{K}^{3}}{f_{K}^{3}}+c_{4}^{\prime \prime} \frac{m_{K}^{4}}{f_{K}^{4}} .
\end{aligned}
$$

In Fig. 2 our results together with the fits are presented.

\section{3 $D-\bar{D}^{*}$}

In the $D-\bar{D}^{*}, I=1$ channel, there is no quark exchange if we ignore the $c \bar{c}$ annihilation. The energy shifts $\Delta E$ at the four light quark masses are all negative which indicates attractive interactions, while the sign of the scattering length at the lowest light quark mass changes as shown in Fig. 3. From the viewpoint of non-relativistic quantum mechanics, a bound state with small binding energy emerges when the sign of the scattering length changes due to increased attraction ${ }^{1}$. We assume that this argument is justified in our case since we are studying the scattering near the threshold. Our data suggests that there is bound state in this channel.

\footnotetext{
${ }^{1}$ See "Modern Quantum Mechanics", Revised Edition, by J. J. Sakurai, §413-415. Note that our definition of the scattering length differs from theirs by a sign.
} 

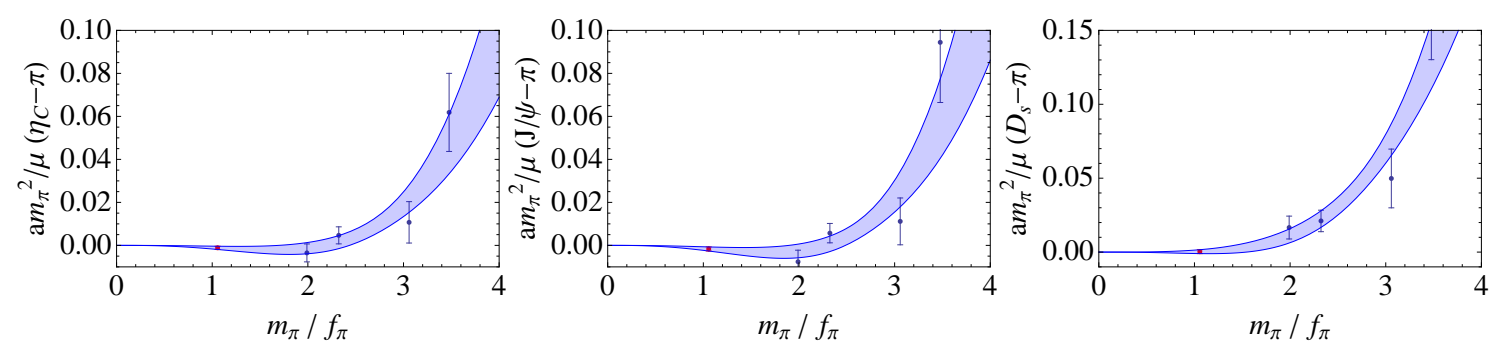

Figure 5: Scattering lengths of $\eta_{c}-\pi, J / \Psi-\pi$ and $D_{s}-\pi$ as functions of $m_{\pi} / f_{\pi}$.

$4.4 D-\pi$

The s-wave scattering length of $D-\pi$ at next-to-leading order chiral perturbation theory are [13]

$$
\begin{aligned}
& a^{(3 / 2)}=\frac{1}{8 \pi}\left(1+\frac{m_{\pi}}{M_{D}}\right)^{-1}\left(-\frac{m_{\pi}}{f_{\pi}^{2}}+C_{1} \frac{m_{\pi}^{2}}{f_{\pi}^{2}}\right), \\
& a^{(1 / 2)}=\frac{1}{8 \pi}\left(1+\frac{m_{\pi}}{M_{D}}\right)^{-1}\left(2 \frac{m_{\pi}}{f_{\pi}^{2}}+C_{1} \frac{m_{\pi}^{2}}{f_{\pi}^{2}}\right),
\end{aligned}
$$

where the subscripts denote the isospin.

We didn't calculate the $\mathrm{I}=1 / 2$ channel due to the difficulty arising from the disconnected diagrams. We only calculate the $\mathrm{I}=3 / 2$ channel. The extrapolation formula for this channel is

$$
a \frac{m_{\pi}^{2}}{\mu}=-\frac{1}{8 \pi} \frac{m_{\pi}^{2}}{f_{\pi}^{2}}+\frac{1}{8 \pi} C_{1} \frac{m_{\pi}^{3}}{f_{\pi}^{3}}
$$

where $\mu=\frac{m_{D} m_{\pi}}{m_{D}+m_{\pi}}$. Fig.4 shows this extrapolation. The fitted scattering length is -0.073(2). Substitute the fitted $C_{1}$ into Eq .4.8, we predict the scattering length of $D-\pi$ isospin-1/2 to be $0.104(2)$.

$4.5 \eta_{c}-\pi, J / \Psi-\pi$ and $D_{s}-\pi$

The s-wave scattering length of a heavy hadron with the pion is given by [14]

$$
a_{0}^{H-\pi}=-\left(1+\frac{m_{\pi}}{M_{H}}\right)^{-1} \frac{m_{\pi}}{8 \pi f_{\pi}^{2}}\left[I(I+1)-I_{H}\left(I_{H}+1\right)-2\right]+\mathscr{O}\left(m_{\pi}^{2}\right)
$$

For $\eta_{c}-\pi, J / \Psi-\pi$ and $D_{s}-\pi$ channels, the first term in Eq.4.9 vanishes. We do the chiral extrapolation using

$$
a \frac{m_{\pi}^{2}}{\mu}=c_{3} \frac{m_{\pi}^{3}}{f_{\pi}^{3}}+c_{4} \frac{m_{\pi}^{4}}{f_{\pi}^{4}}
$$

where $\mu=\frac{m_{H} m_{\pi}}{m_{H}+m_{\pi}}$. Fig.4.5 shows the extrapolations the scattering lengths of $\eta_{c}-\pi, J / \Psi-\pi$ and $D_{s}-\pi$. The extrapolated scattering lengths all agree with zero within statistical errors. 


\subsection{Conclusions}

The most interesting result of this work is the sign change of the scattering length of $D-$ $\overline{D^{*}}(I=1)$ channel, suggesting that it is very likely that there is a loosely bound state in this channel. We also calculate the scattering lengths of the charmonia and charmed mesons $\left(\eta_{c}, J / \Psi, D\right.$ and $\left.D_{s}\right)$ with the light hadrons $(N, \pi$ and $K)$. In the charmonia-N channels, we see attractive interactions. These channels are pretty noisy as shown in Fig. 1. The large error bars of the scattering lengths come from the inverse of $S$-function, defined in Eq. 3.3, evaluated near a pole. Coupled channel effects may also contribute to the noise. Finally we observe relatively strong attractive interaction in $D-K(I=1)$ channel. In the future, we are going to explore the use of $\chi \mathrm{PT}$ to perform the light quark extrapolation. By doing this, we can get the LECs and predict the scattering lengths of some channels which are interesting but we are unable to calculate due to the disconnected diagrams, such as $D-K(I=0)$ and $D-\pi(I=1 / 2)$. We will also study the coupled channel effects.

\subsection{Acknowledgement}

I thank the NPLQCD and LHP collaborations for sharing their light and strange propagators. I also thank Andre Walker-Loud and Huey-Wen Lin for important contributions to this work. Calculations were performed using the Chroma software suite, on computer clusters at Jefferson Laboratory (USQCD SciDAC supported) and the College of William and Mary (Cyclades cluster supported by the Jeffress Memorial Trust grant J-813). This work was supported in part by Jefferson Science Associates under U.S. DOE Contract No. DE-AC05-06OR23177 and in part by DOE grant DE-FG02-07ER41527.

\section{References}

[1] S. R. Beane, K. Orginos, and M. J. Savage, Int. J. Mod. Phys. E17, 1157-1218 (2008).

[2] K. Yokokawa, S. Sasaki, T. Hatsuda, and A. Hayashigaki, Phys. Rev. D74, 034504 (2006)

[3] G.-Z. Meng et al., Phys.Rev.D80, 034503 (2009)

[4] S.-L. Zhu, Int. J. Mod. Phys, E17, 283 (2008).

[5] S. J. Brodsky, I. A. Schimidt, and G. F. de Teramond, Phys. Rev. Lett. 64, 1011 (1990).

[6] M. E. Luke, A. V. Manohar, and M. J. Savage, Phys. Lett. B288, 355 (1992).

[7] S. J. Brodsky and G. A. Miller, Phys. Lett. B412, 125 (1997).

[8] L. Maiani and M. Testa, Phys. Lett. B245, 585 (1990).

[9] M. Lüscher, Commun. Math. Phys. 105, 153 (1986).

[10] M. Lüscher, Nucl. Phys. B354, 531 (1991).

[11] L. Liu, H.-W. Lin, K. Orginos, and A. Walker-Loud, arXiv:0909.3294v2 [hep-lat]

[12] F.-K. Guo, C. Hanhart, and Ulf-G Meißner, Eur. Phys. J. A40, 171-179 (2009).

[13] Y.-R. Liu, X. Liu, and S.-L. Zhu, Phys. Rev. D79, 094026 (2009).

[14] S. Weinberg, Phys. Rev. Lett. 17, 616 (1966). 